\title{
CAUSALIDAD O CONCURRENCIA, RELACIONES ENTRE CAMBIOS AMBIENTALES Y SOCIALES EN LOS CAZADORES RECOLECTORES DURANTE LA TRANSICIÓN ENTRE EL HOLOCENO MEDIO Y TARDÍO (COSTA DEL SEMIÁRIDO DE CHILE)
}

\author{
CAUSALITY OR CO-OCCURRENCE, RELATIONS BETWEEN ENVIRONMENTAL \\ AND SOCIAL CHANGE AMONG HUNTER GATHERERS DURING MID TO LATE \\ HOLOCENE TRANSITION (SEMIARID COAST OF CHILE)
}

César A. Méndez M. ${ }^{1}$ y Donald G. Jackson . $^{1}$

\begin{abstract}
Investigaciones paleoambientales conducidas en el litoral del semiárido de Chile ( $\left.31^{\circ} 50^{\prime} \mathrm{S}\right)$ han revelado una interesante contemporaneidad en la transición entre fases de aridez a humedad, y distintas intensidades de ocupación humana del área. Si bien las variaciones medioambientales son contemporáneas a cambios en los patrones de asentamiento y movilidad, éstas no explican completamente las modificaciones observadas en el registro arqueológico. En este trabajo se discuten los patrones de asentamiento y movilidad durante el Holoceno Medio y Tardío, periodo mejor documentado por el registro polínico local (post 6.100 años cal. a.p.), en función de variables contextuales, dataciones absolutas, registro zooarqueológico y los conjuntos de artefactos. El objetivo constituye evaluar la "causalidad" o "concurrencia" medioambiental como factores responsables para los cambios en las conductas de los cazadores recolectores del área de estudio. Finalmente, se sostiene que en el cambio cultural debieron operar diversas variables de manera integrada.
\end{abstract}

Palabras claves: cazadores recolectores y medio ambiente, movilidad, respuestas sociales.

Palaeoenvironmental research along the Chilean semiarid coast line (31 $50^{\circ}$ 'S) has revealed interesting contemporaneous trends in the transition from arid to humid phases, and differences in intensity of human occupation in the area. Even though environmental variation is contemporary to changes in settlement and mobility patterns, these do not fully explain modifications observed in the archaeological record. In this paper, we discuss settlement patterns and mobility during Mid to Late Holocene, a well-documented period from the local pollen record (post 6,100 cal. years B.P.), on the basis of contextual variables, radiocarbon chronology, zooarchaeological record and artifact assemblages. Our objective is to assess environmental "causality" versus "co-occurrence" as factors responsible for changes in hunter gatherers' conduct in the study area. Finally, we propose cultural change as the result of several integrated variables operating contemporaneously.

Key words: Hunter gatherers and environment, mobility, social responses.

Las investigaciones arqueológicas referidas a la relación entre hombre y medio ambiente tienen larga data en la arqueología (Dincauze 2000). Recurrente ha sido (y es) la tendencia a caer en lo que ha sido disciplinariamente denominado como determinismo ambiental, o la producción de explicaciones causales al actuar humano desde un acontecimiento de fluctuación particular o conjunto de condiciones climático-ambientales dadas. Lo cierto es que la totalidad de los grupos humanos existen e interactúan con el medio ambiente, afectándose mutuamente (Mena 1996), incluso con distintos grados de vulnerabilidad y dependencia (Messerli et al. 2000). Particularmente, aquellas poblaciones de modo de vida cazador recolector han sido integradas a modelos de interpretación con un estrecho vínculo a la variable ambiental (Kelly 1995), probablemente porque sus decisiones económicas se sustentan en una dependencia más extractiva, que productiva, sobre la estructura de los recursos.

La arqueología de la costa del Pacífico sudamericano no ha estado exenta a la generación de modelos que impliquen relaciones entre el hombre

1 Departamento de Antropología, Facultad de Ciencias Sociales, Universidad de Chile. Ignacio Carrera Pinto 1045, Ñuñoa, Santiago.cmendezm@uchile.cl; djackson@uchile.cl 
y un medio ambiente cambiante (Jerardino 1995; Reitz y Sandweiss 1996; Sandweiss et al. 1996, 2001; Sandweiss 2003). En Chile, y particularmente en el territorio semiárido litoral, destacan modelos de interpretación con una fuerte raigambre ambiental, entre los cuales se subrayan, ya sean condiciones locales particulares para el asentamiento de grupos paleoindios (Núñez et al. 1987; 1994), o bien, respuestas culturales a condiciones ambientales áridas en la forma del desarrollo de sistemas de asentamiento organizado y alta movilidad a distancia (Jackson 2002).

Una de las fluctuaciones ambientales de mayor discusión en la actualidad corresponde a la transición entre el Holoceno Medio y Tardío, vale decir, el cambio desde el último mayor pulso ambiental árido y cálido, al advenimiento de las condiciones ambientales contemporáneas más húmedas y frías. Muchos trabajos coinciden en que este momento, que dista de ser homogéneo, no se inicia de forma contemporánea en todas las regiones y se caracteriza más bien por una serie de modificaciones climáticas menores, aunque significativas, que incluyen cambios percibidos en archivos vegetacionales (Jenny et al. 2002; Maldonado y Villagrán 2002; Villa-Martínez et al. 2003) y geoarqueológicos (Sandweiss et al. 2001), entre otros.

Recientemente, Maldonado y Villagrán (2002) propusieron para la zona meridional del norte semiárido una correlación entre la intensidad de ocupación arqueológica del litoral y los distintos momentos ambientales identificados en columnas polínicas de bosques pantanosos.

Our results at Nagué site show two arid
phases, with a prevalence of xeric, open
vegetation occurring between $\sim 6100$ and
4200 cal yr B.P. and again between $\sim 1800$
and 1300 cal yr B.P. These time periods
are coherent with the chronology for oc-
casional human occupation of the area.
In contrast, the first phase of forest deve-
lopment in Nagué site ( 4400 and 1800
cal yr B.P.), coincides with the period of
more permanent human settlement on the
area. (Maldonado y Villagrán $2002: 136)$.

La información arqueológica actualmente manejada para el área de estudio (Los Vilos, Provincia del Choapa, IV Región) respalda las propuestas de los autores citados. Sin embargo, al tra- tarse de una investigación paleoambiental, su énfasis se centra en la exposición de dichos cambios y su eventual relación con las ocupaciones humanas, y no viceversa. En este sentido, se enuncia la contemporaneidad y correlación entre medio ambiente e intensidad de ocupación regional, sugiriéndose implícitamente que sería el cambio ambiental el motor tras el cambio en el patrón de asentamientos observado prehistóricamente. En el presente trabajo nos proponemos invertir el énfasis de la discusión, pretendiendo tomar los datos ambientales en función de la generación de un modelo interpretativo, que relacione a los grupos humanos y su medio ambiente fluctuante. En este sentido se pretende precisar los efectos del cambio ambiental entre los grupos de cazadores recolectores del Holoceno Medio y Tardío, tomando en consideración diversas líneas de evidencia arqueológica que enriquezcan la argumentación, especialmente aquellas variantes del asentamiento y la movilidad. Para su caracterización se integrarán los contextos arqueológicos, condicionantes del emplazamiento de los campamentos, funcionalidad de los sitios, recursos consumidos y conjuntos artefactuales, todos mediados por una cantidad importante de dataciones radiocarbónicas restringidas a un área menor de $25 \mathrm{~km}^{2}$. La propuesta considera que, tras un periodo de aridez en donde grupos desde la vertiente oriental de la cordillera accedieron de forma pautada estacional a la costa, sobreviene una radicación permanente (durante el año) bajo una activa movilidad residencial a lo largo del eje costero. El advenimiento de condiciones húmedas en coincidencia con este acontecimiento no lo explica, sino que corresponde a uno de tantos otros factores que concurren en el cambio. El modelo, si bien se desarrolla en una región específica, debiera ser eventualmente contrastado en otras latitudes.

\section{Área de Estudio y Paleoambiente}

La zona costera de Los Vilos ( $31^{\circ} 50^{\prime} \mathrm{S}$ ) se ubica en el segmento meridional del norte semiárido de Chile. Esta área se caracteriza por escasas precipitaciones y está dominada por vegetación abierta de Jaral Costero. Es interrumpida ocasionalmente por quebradas con bosques pantanosos desde los $30^{\circ} \mathrm{S}$ hacia el sur (Maldonado y Villagrán 2002), los que se alimentan principalmente de las aguas subterráneas y en menor medida incorporan 
aquellas precipitadas (Varela 1981). Esta vegetación de matorral se compone fundamentalmente de arbustos y hierbas mesófitas, extendiéndose en el litoral costero entre los $31^{\circ}$ y $34^{\circ} \mathrm{S}$ (Donoso 1981).

El clima del área de estudio corresponde a una estepa con nubosidad abundante (BSn-Fuenzalida 1965), con temperaturas promedio de $14^{\circ} \mathrm{C}$, rangos máximos de humedad en época estival (entre $84 \%$ y $71 \%$ ) y precipitaciones medias de $262 \mathrm{~mm}$, que se concentran principalmente en la estación invernal (Varela 1981). Propiamente, en el litoral de Los Vilos se registran las variedades arbustivas Bahia ambrosioides, Baccharis concava y Puya venusta y herbáceas Anasomeria litoralis, Lobelis polypilla y Fucsia lycoides; mientras que en las planicies litorales dominan Haplappopus foliosus, Eulychnia costanea, Franseria sp., Frankenia sp. y Calandrina sp. (Seguel et al. 1995). En los bosques pantanosos, por su parte, dominan los árboles Luma chequen, Drimys winteri y Escallonia revoluta (Maldonado y Villagrán 2002).

Geológicamente, el área de Los Vilos se caracteriza por tres sectores de rocas fundamentales (septentrional, central y meridional), los que son afectados por cuatro sistemas de fallas, las que definen una cuenca con relleno de sedimentos cuaternarios (graben central, Varela 1981). Las trasgresiones marinas formaron un sistema de tres terrazas marinas (alta, intermedia y baja) de distintos rangos de altura (140 a $120 \mathrm{msm}, 40$ a 25 msm y 7 a $6 \mathrm{msm}$, respectivamente), con diferentes edades relativas, aunque todas cuaternarias (antiguo, medio y reciente-holocénico, respectivamente) (Varela 1981). Finalmente, el área posee un sistema de depósitos de dunas fósiles parcialmente removilizados por acción eólica (Paskoff 1970; Varela 1981). Estas últimas, en conjunto con la terraza marina intermedia, resultaron fundamentales para el asentamiento prehistórico de la zona, emplazando a la mayoría de los contextos arqueológicos estudiados (Jackson 2002; Méndez y Jackson 2004).

Durante la segunda mitad del Holoceno, el clima entre los $27^{\circ}$ y $33^{\circ} \mathrm{S}$ puede ser distinguido como seco, con variaciones menores frías y húmedas y acción erosiva sobre los suelos formados anteriormente (Viet 1993). Estas variaciones, especialmente la transición entre el Holoceno Medio y Tardío, caracterizan el advenimiento de las condiciones climáticas actuales. No obstante, los métodos, ar- chivos ambientales y muestras para establecer este cambio no son necesariamente coincidentes y operan a distintas escalas, siendo necesaria una aproximación desde múltiples indicadores (Grosjean et al. 2003). Por estas razones, para la realización de una síntesis paleoambiental se consideró la información disponible a escala regional entre $\operatorname{los} 30^{\circ} \mathrm{y}$ $34^{\circ} \mathrm{S}$ (Figuras 1 y 2 ).

Condiciones de mayor humedad han sido establecidas a partir de eventos de escurrimiento de laderas ausentes hasta los 5.700 años cal. a.p., y frecuentes posterior a los 3.200 años cal. a.p. en los núcleos extraídos de laguna Aculeo $\left(34^{\circ} \mathrm{S}\right.$, Jenny et al. 2002). El cambio climático se manifestó a partir del reemplazo de polen terrestre (dominante durante el Holoceno Medio) por taxones acuáticos, como también en la modificación experimentada por las diatomeas, planteándose consecuentemente un aumento en la actividad de ENSO (Jenny et al. 2002). En la costa de Quintero (32 $2^{\circ} 50^{\prime}$ 'S) el reemplazo sedimentario hacia los 4.000 años cal. a.p. permitió fechar el advenimiento de una fase pluvial (sitio Quintero I, Villagrán y Varela 1990), mientras que el reemplazo vegetacional en el registro polínico, hacia los 4.500 años cal. a.p., sugiere condiciones climáticas más húmedas que el momento inmediatamente anterior (Quintero II, Villa-Martínez y Villagrán 1997). Evaluaciones de isótopos de oxígeno $\left(\delta^{18} \mathrm{O}\right)$ realizadas sobre moluscos arqueológicos detectaron el mencionado cambio ambiental hacia los $\sim 3.800$ años cal. a.p. a partir de la observación de temperaturas marinas más frías (3330' S), para que luego se iniciase un alza moderada hacia fechas de los $\sim 2.000$ años cal. a.p. (Falabella et al. 1991). Por su parte, Lamy y colaboradores (1999), a partir del análisis de deposición sedimentaria marina en las desembocaduras de los ríos Aconcagua ( $\left.33^{\circ} \mathrm{S}\right)$ y Maipo $\left(33^{\circ}\right.$ 60 'S), establecen el cambio hacia mayor humedad hacia los $\sim 4000$ años cal. a.p. Una alta tasa de sedimentación y cambios en la composición química y características de los sedimentos son los argumentos que permitirían pensar en una menor alimentación de la fuente de la Cordillera de los Andes e incremento de material de la Cordillera de la Costa, en conjunto con un mayor desgaste químico en la última (Lamy et al. 1999).

Un total de seis fechados radiocarbónicos (Figura 1$)^{1}$ sobre dos columnas de polen fósil (Ñagué I y III) obtenidas en el bosque pantanoso de Ñagué (Figuras 2 y 3 ) permitieron la generación de una 


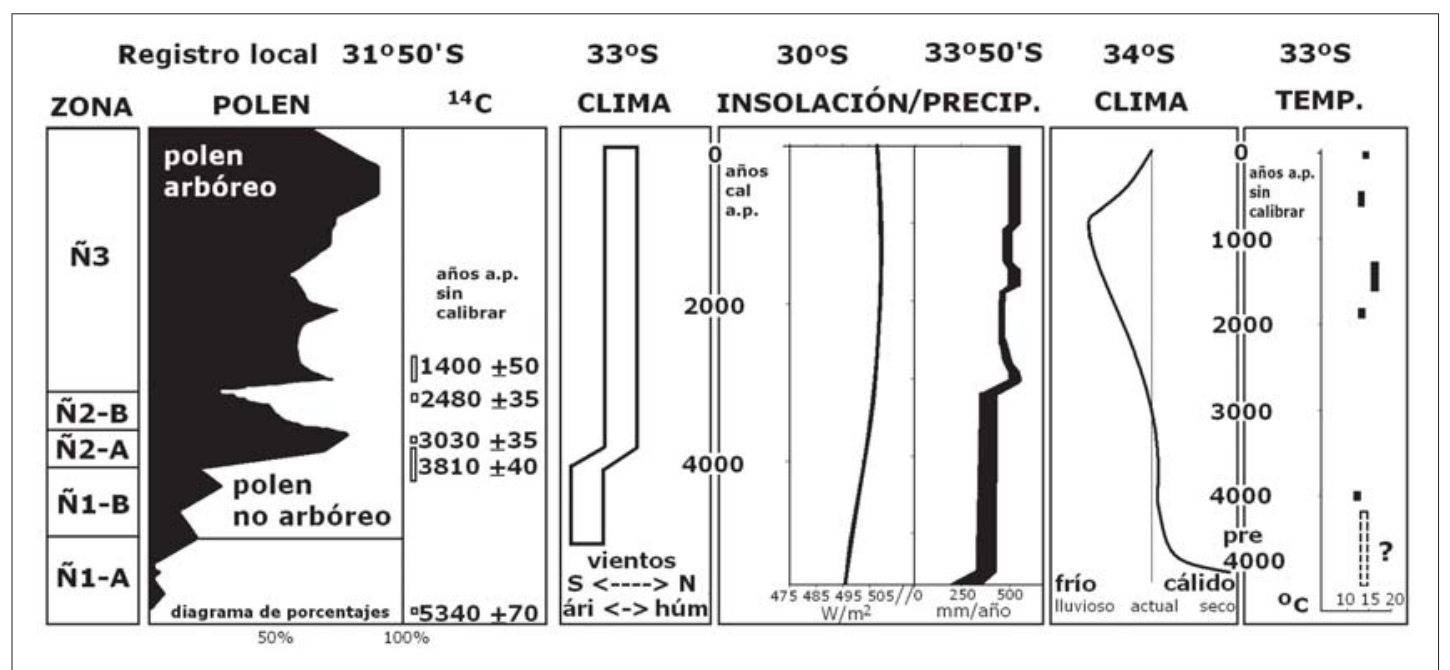

Figura 1. Panorama ambiental para la transición entre el Holoceno Medio y Tardío entre los $30^{\circ}$ y $34^{\circ}$ S. Perfil polínico Ñagué I (31 50'S), zonas, resumen de porcentajes, dataciones sin calibrar y sus posiciones estratigráficas (Maldonado y Villagrán 2002). Reconstrucción climática $\left(33^{\circ} \mathrm{S}\right)$, humedad relativa y dirección de los vientos sobre dataciones calibradas (Lamy et al. 1999). Insolación estival (mes de diciembre, $30^{\circ} \mathrm{S}$ ) en el hemisferio sur sobre dataciones calibradas (Berger y Loutre 1991, en Jenny et al. 2003). Precipitación estimada para la Laguna Aculeo (3350'S) sobre dataciones calibradas (Jenny et al. 2003). Interpretación climática de la transición entre Holoceno medio y tardío sobre dataciones sin calibrar para la zona de Taguatagua $\left(34^{\circ} \mathrm{S}\right.$; Varela 1976, Núñez et al. 1987). Paleotemperaturas marinas a partir de análisis de $\delta^{18} \mathrm{O}$ sobre dataciones sin calibrar para el litoral de Las Cruces (33우 Falabella et al. 1991).

Mid to Late Holocene environmental overview between $30^{\circ}$ and $34^{\circ} \mathrm{S}$. Pollen profile of Nagué I (31 $50^{\circ}$ 'S), zones, summarized percentages, uncalibrated dates and their stratigraphic positions (Maldonado and Villagrán 2002). Climate reconstruction ( $33^{\circ} S$ ), relative humidity and wind direction on calibrated dates (Lamy et al. 1999). Southern hemisphere summer insolation (December, $30^{\circ} \mathrm{S}$ ) on calibrated dates (Berger and Loutre 1991, in Jenny et al. 2003). Estimated precipitation for Laguna Aculeo (33 $50^{\prime}$ 'S) on calibrated dates (Jenny et al. 2003). Mid to Late Holocene climate interpretation on uncalibrated dates for the Taguatagua area $\left(34^{\circ} \mathrm{S}\right.$; Varela 1976, Núñez et al. 1987). $\delta^{18}$ O marine paleotemperatures on uncalibrated dates for Las Cruces coastline (33 ${ }^{\circ}$; Falabella et al. 1991).

secuencia climática para el Holoceno superior en la región meridional del norte semiárido (Maldonado y Villagrán 2002). Un esquema sintético se expone en la Tabla 1. Los autores plantean que previo a los $\sim 4.200$ años cal. a.p. prevalecieron condiciones más áridas que en la actualidad, permitió el dominio de vegetación abierta y herbácea (taxones xerófitos) en sectores hoy distinguidos por vegetación arbórea (Maldonado y Villagrán 2002). El incremento de precipitaciones en la misma fecha, consecuentes con el advenimiento de condiciones más húmedas, permitió el alza de los niveles de agua subterránea estableciéndose los mencionados bosques pantanosos (Maldonado y Villagrán 2002). Investigaciones anteriores en la zona (Quereo) habían advertido este cambio hacia fechas de 3.200 cal. a.p. (Núñez et al. 1994; Villagrán y Varela 1990). Posteriores fluctuaciones climáticas menores, aunque con una significativa fase de aridez entre los $\sim 1.800$ y 1.300 años cal. a.p., devinieron en la conformación del clima actual (Maldonado y Villagrán 2002).
Por su parte, gravitante al tema de las ocupaciones litorales resulta la discusión de la formación de terrazas marinas, área privilegiada para el asentamiento humano prehistórico. En vistas de la isostasia relativamente estable que caracteriza a esta franja costera, es posible atribuir una edad del Pleistoceno Final a la terraza marina intermedia (Paskoff 1970). J. Montané (1964) en su pionero trabajo geoarqueológico propone una edad máxima para esta última terraza de 2.000 años a.p., estableciéndose los límites del rango para las ocupaciones en esta formación. Investigaciones posteriores establecieron que la elevación del nivel marino holocénico habría concluido hacia los $\sim 6.800$ años cal. a.p. (medido en los $30^{\circ} 15^{\prime} \mathrm{S}$ en un rango entre 3 y $5 \mathrm{msm}$ actual), para iniciarse después una regresión hacia los niveles actuales, ya patente hacia los 5.000 y 4.000 años cal. a.p. (Ota y Paskoff 1993). Con todo, información climática diversa en una amplia escala, datos polínicos locales de alta fidelidad y un importante desarrollo en torno al estudio de las formaciones geomorfológicas litorales, 


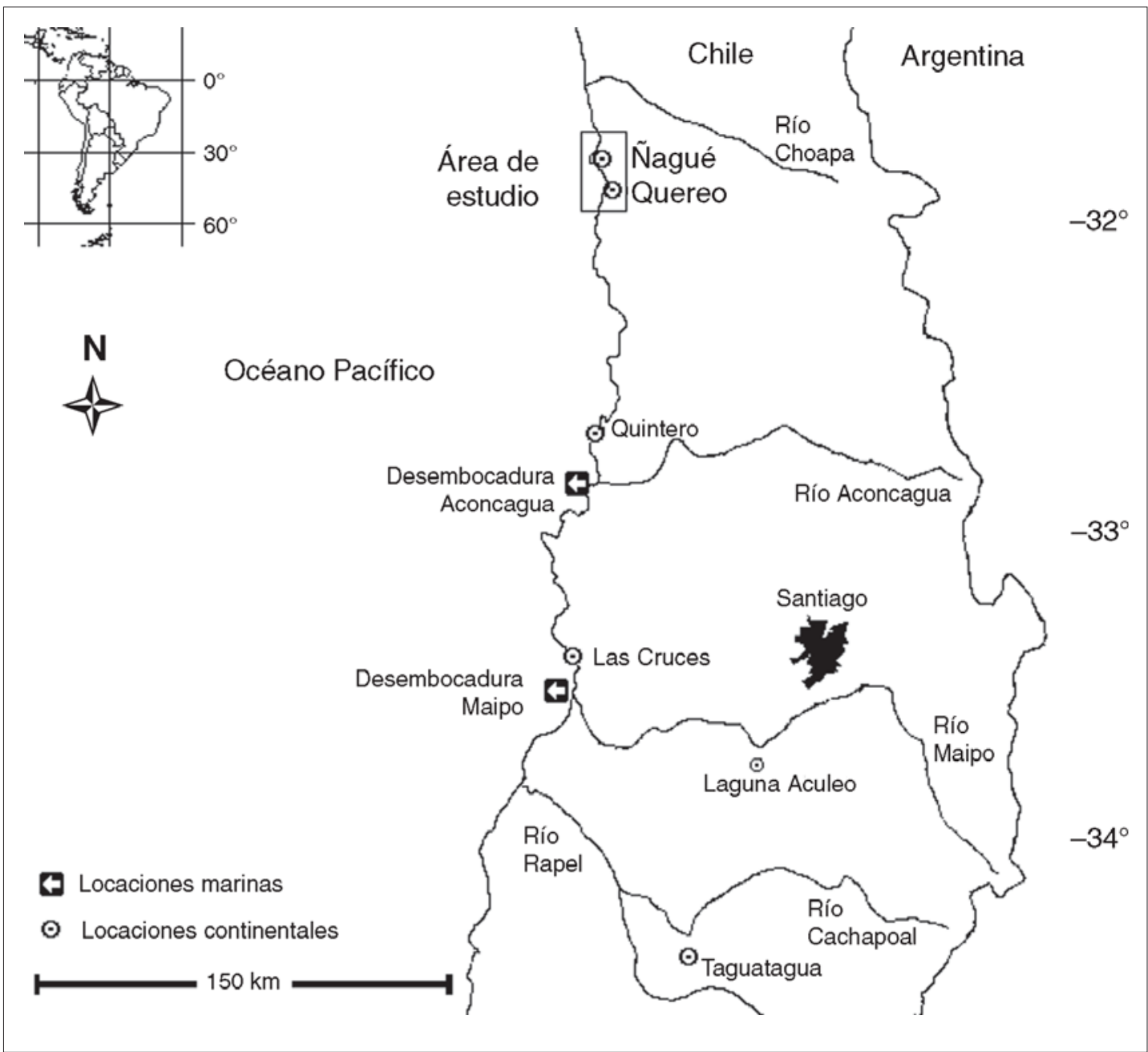

Figura 2. Locaciones de información ambiental mencionadas en el texto. Environmental sites mentioned in the text.

es posible visualizar un panorama ambiental de especial precisión, el cual consiente evaluar modelos de la relación hombre y su medio.

\section{Ocupaciones Humanas del Litoral de}

\section{Los Vilos Durante el Holoceno Medio y Tardío}

La contemporaneidad entre el cambio ambiental ocurrido hace 4.000 años cal. a.p. y la modificación en los patrones arqueológicos (formalmente observados e inferidos) en el área de Los Vilos resulta bastante sugerente, es especial, en vista que ambas alteraciones muestran ser bastante marcadas. Cazadores recolectores, que alguna vez transitaron de forma trashumante entre amplios espa- cios costeros e interiores, y muy probablemente integrando la vertiente oriental de la cordillera de los Andes (Jackson 2002), se establecen en territorio litoral, con una consecuente modificación en todas las manifestaciones producto de su acción (Méndez 2003; Méndez y Jackson 2004).

Entre los $\sim 7.700$ y 4.200 años cal. a.p. (Tabla 2) el área de estudio fue habitada por grupos de cazadores recolectores, cuyos patrones formales han permitido generar un modelo hipotético de ocupación estacional del área (Jackson 2002). Dos tipos de yacimientos claramente diferenciados caracterizan a los campamentos (Jackson et al. 1997, Figura 3a). Los más ubicuos y frecuentes a lo largo del eje costero corresponden a conchales de 


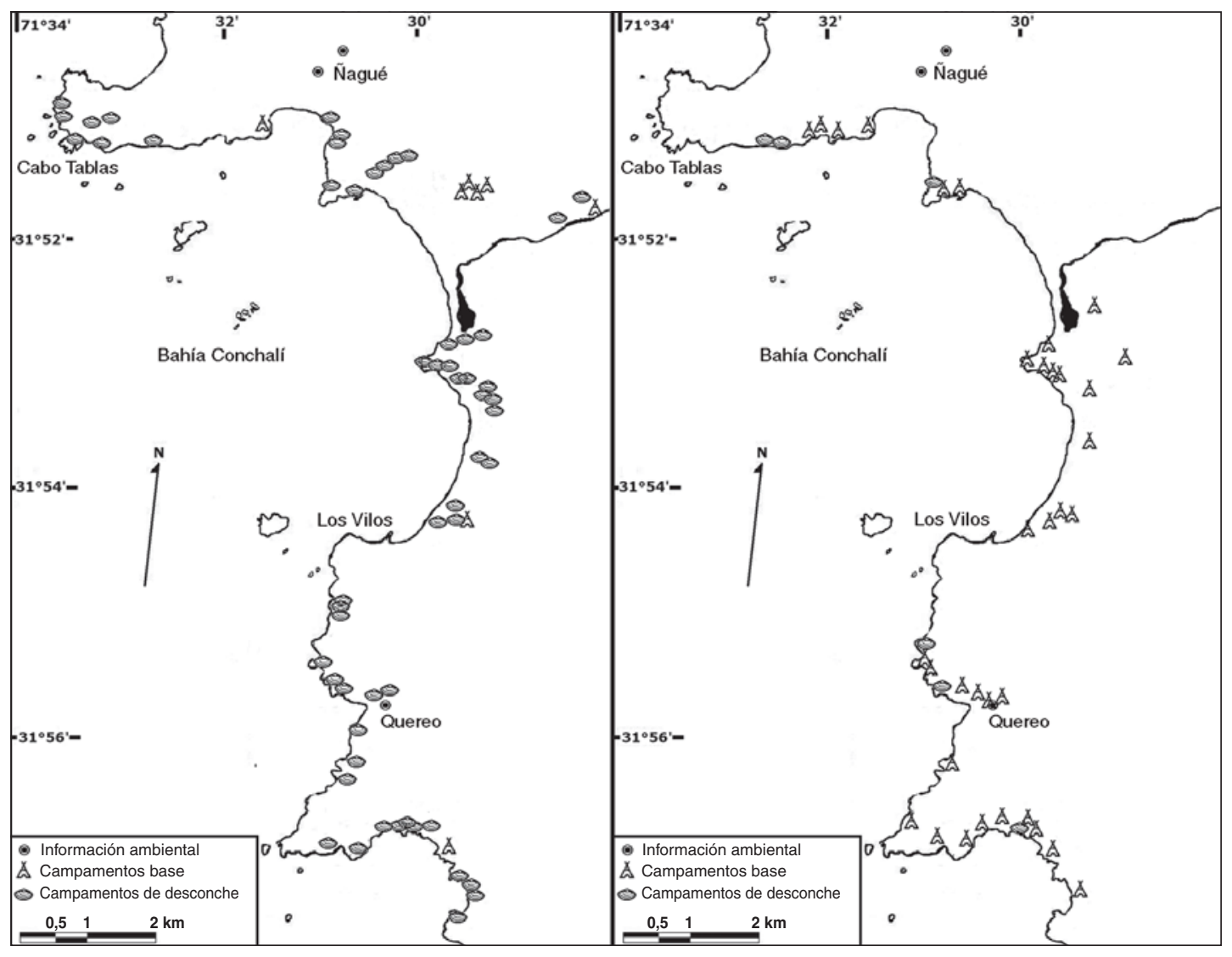

Figura 3. Mapa del área de estudio. (a) Derecha: panorama arqueológico hacia el Holoceno Medio ( 7.700 a 4.200 años cal. a.p.) adaptado de la información vertida en Jackson 2002. (b) Izquierda: panorama arqueológico hacia la primera mitad del Holoceno Tardío ( 3.800 a 2.000 años cal. a.p.).

Map of the study area. (a) Right: Mid Holocene archaeological record ( 7,700 to 4,200 cal. years B.P.) adapted from Jackson 2002. (b) Left: Archaeological record ( 3,800 to 2,000 cal. years B.P.) toward the first half of the Late Holocene.

Tabla 1. Formaciones vegetales dominantes obtenidas en los registros polínicos del bosque pantanoso de Ñagué (Maldonado y Villagrán 2002).

Dominant vegetation formations obtained from pollen records at Ñagué swamp forest (Maldonado and Villagrán 2002).

\begin{tabular}{|c|c|c|}
\hline Columna/zona & Años cal. a.p. & Vegetación dominante \\
\hline $\mathrm{I} / \tilde{\mathrm{N}}-1 \mathrm{~A}$ & $6.100-5.300$ & No arbórea \\
\hline $\mathrm{I} / \tilde{\mathrm{N}}-1 \mathrm{~B}$ & $5.300-4.200$ & No arbórea (incremento de vegetación arbórea hacia el final) \\
\hline $\mathrm{I} / \tilde{\mathrm{N}}-2 \mathrm{~A}$ & $4.200-3.200$ & Arbórea (incremento marcado) \\
\hline $\mathrm{I} / \tilde{\mathrm{N}}-2 \mathrm{~B}$ & $3.200-2.600$ & No arbórea (decrece vegetación arbórea) \\
\hline $\mathrm{I} / \tilde{\mathrm{N}}-3$ & $1.800-0$ & Arbórea (fluctuaciones menores) \\
\hline $\mathrm{III} / \tilde{\mathrm{N}}-1$ & & No arbórea \\
\hline III/Ñ -2 A & & Arbórea (incremento marcado) \\
\hline III/N-2 B & & No arbórea (decrece vegetación arbórea) \\
\hline III/Ñ -2 C & & No arbórea (incremento) \\
\hline III/Ñ-3 & & Arbórea (incremento) \\
\hline
\end{tabular}


Tabla 2. Dataciones para la secuencia arqueológica ocupacional del Holoceno Medio y Tardío (1) Román y Jackson 1998, (2) Jackson et al. 2004, (3) Jackson 2003, (4) Jackson et al. 1996, (5) Jackson y Ampuero 1993, (6) Jackson 2002, (7) Seguel et al. 1995, (8) Pimentel 2001, (9) Méndez et al. 2004, (10) Barrera y Belmar 2000, (11) Artigas 2001, (12) Núñez et al. 1994, (13)

Méndez y Jackson 2002, (14) Méndez 2002a. Todas las dataciones fueron calibradas con el programa Ox Cal vers. 3.5, con información ambiental de Stuiver et al. 1998, sin corrección por efecto reservorio local.

Mid to Late Holocene dates for the archaeological occupational sequence (1) Román and Jackson 1998, (2) Jackson et al. 2004, (3) Jackson 2003, (4) Jackson et al. 1996, (5) Jackson and Ampuero 1993, (6) Jackson 2002, (7) Seguel et al. 1995, (8) Pimentel 2001, (9) Méndez et al. 2004, (10) Barrera and Belmar 2000, (11) Artigas 2001, (12) Núñez et al. 1994, (13) Méndez and Jackson 2002, (14) Méndez 2002a. All dates were calibrated with Ox Cal vers. 3.5 with environmental information from Stuiver et al. 1998, without local reservoir effect correction.

\begin{tabular}{|c|c|c|c|c|}
\hline $\begin{array}{l}\text { Yacimiento/ ocupación } \\
\text { (referencia) }\end{array}$ & Función & Método/muestra & $\begin{array}{l}\text { Resultado } \\
\text { años a.p. }\end{array}$ & $\begin{array}{l}\text { Calibración } 2 \text { sigma } \\
\max \text { (porcentaje) min } \\
\text { años a.p. }\end{array}$ \\
\hline Agua Amarilla LV. 166 (1) & Campamento base & TL en roca de fogón & & $6.800 \pm 500$ \\
\hline Agua Amarilla LV. 166 (1) & Campamento base & TL en roca de fogón & & $6.775 \pm 500$ \\
\hline Punta Chungo LV. 046A V (2) & Campamento logístico & $\mathrm{C}^{14}$ conv. valva & $6.710 \pm 60$ & $7.680(0,99) 7.460$ \\
\hline Agua Amarilla LV. 166 (3) & Campamento base & $\mathrm{C}^{14}$ conv. valva & $6.700 \pm 60$ & $7.680(0,98) 7.460$ \\
\hline Agua Amarilla LV. 166 (1) & Campamento base & TL en roca de fogón & & $6.600 \pm 300$ \\
\hline Agua Amarilla LV. 166 (1) & Campamento base & TL en roca de fogón & & $6.500 \pm 300$ \\
\hline Agua Amarilla LV. 166 (1) & Campamento base & TL en roca de fogón & & $6.480 \pm 325$ \\
\hline Quereo Norte LV.036 (4) & Campamento logístico & $\mathrm{C}^{14}$ conv. valva & $6.030 \pm 80$ & $7.030(0,95) 6.670$ \\
\hline Los Cerrillos LV. 077 (5) & Campamento logístico & $\mathrm{C}^{14}$ conv. valva & $5.820 \pm 60$ & $6.760(1,00) 6.470$ \\
\hline Punta Chungo LV. 046B V (6) & Campamento logístico & $\mathrm{C}^{14}$ conv. valva & $5.700 \pm 80$ & $6.670(0,99) 6.300$ \\
\hline Agua Amarilla LV. 099 (7) & Campamento base & $\mathrm{C}^{14}$ conv. valva & $5.480 \pm 70$ & $6.410(0,96) 6.170$ \\
\hline Punta Chungo LV. 046B IV (6) & Campamento logístico & $\mathrm{C}^{14}$ conv. valva & $5.480 \pm 70$ & $6.410(0,96) 6.170$ \\
\hline Agua Amarilla LV. 164 (8) & Campamento base & $\mathrm{C}^{14}$ conv. valva & $5.420 \pm 80$ & $6.350(0,97) 5.990$ \\
\hline Punta Chungo LV. 046B III (6) & Campamento logístico & $\mathrm{C}^{14}$ conv. valva & $5.270 \pm 70$ & $6.210(0,97) 5.900$ \\
\hline Punta Chungo LV. 046A III (2) & Campamento logístico & $\mathrm{C}^{14}$ conv. valva & $5.010 \pm 80$ & $5.930(1,00) 5.590$ \\
\hline Punta Chungo LV. 046B II (6) & Campamento logístico & $\mathrm{C}^{14}$ conv. valva & $3.960 \pm 70$ & $4.650(1,00) 4.150$ \\
\hline La Cachina LV. 114 (9) & Campamento residencial & TL en roca de fogón & & $4.020 \pm 230$ \\
\hline Punta Chungo LV. 046B I (6) & Campamento residencial & $\mathrm{C}^{14}$ conv. valva & $3.390 \pm 70$ & $3.840(1,00) 3.460$ \\
\hline Paso Inferior Conchalí LV. 034 (10) & Campamento residencial & $\mathrm{C}^{14}$ conv. valva & $3.280 \pm 50$ & $3.640(1,00) 3.380$ \\
\hline P. Nagué - cabo Tablas LV. 203 (11) & Campamento residencial & $\mathrm{C}^{14}$ AMS carbón & $3.160 \pm 60$ & $3.480(1,00) 3.240$ \\
\hline Punta Chungo LV. 046A I (2) & Campamento residencial & $\mathrm{C}^{14}$ conv. valva & $2.770 \pm 60$ & $3.000(1,00) 2.750$ \\
\hline Quereo III LV. 066 (12) & Campamento residencial & $\mathrm{C}^{14}$ conv. carbón & $2.475 \pm 90$ & $2.760(1,00) 2330$ \\
\hline Quereo III LV. 066 (12) & Campamento residencial & $\mathrm{C}^{14}$ conv. carbón & $2.420 \pm 95$ & $2.800(0,97) 2.300$ \\
\hline Quereo Norte LV. 067 (13) & Campamento residencial & $\mathrm{C}^{14} \mathrm{AMS}$ valva & $2.290 \pm 50$ & $\begin{array}{l}2.360(0,45) 2.290 \\
2.280(0,55) 2.150\end{array}$ \\
\hline Quereo Norte LV. 067 (13) & Campamento residencial & $\mathrm{C}^{14} \mathrm{AMS}$ valva & $2.210 \pm 50$ & $2.350(0,99) 2.100$ \\
\hline Punta Penitente LV. 014 (14) & Campamento residencial & TL en roca de fogón & & $2.160 \pm 100$ \\
\hline
\end{tabular}

Concholepas concholepas, cuyo gran tamaño permite sugerir una selectividad económica (Jackson et al. 1996; 1997) en especial si la comparamos a otros periodos (Méndez 2002b). En estos campamentos de corta duración se llevó a cabo un conjunto restringido de tareas enfocadas al procesamiento inicial de los gasterópodos, vale decir, la remoción de su porción comestible, para su posterior transporte hacia los sitios habitacionales (Jackson et al. 1996). Este tipo de sitios son ampliamente reconocidos como logísticos e implican un tipo de movilidad centralizada hacia donde se retorna con el alimento (Binford 1980; Kelly 1995). El conjunto lítico característico corresponde a ins- trumental de procesamiento de manufactura situacional con rocas disponibles inmediatamente y descarte post uso (tajadores, machacadores, percutores y herramientas sin modificaciones intencionales), aunque no exclusivamente destinado al desconche. La gran predictibilidad espacial de los moluscos en el borde litoral (Waselkov 1987) determinó la ubicación de los campamentos en los mismos espacios, generando acumulaciones malacológicas sucesivas, en estratos ocupacionales discretos.

El segundo tipo de yacimiento es aquel denominado campamento base habitacional, centros residenciales a partir de donde se articularían las 
tareas específicas hacia el borde costero. Los campamentos (p.ej. LV. 166) atestiguan estructuración y planificación para su uso espacial interno, característica propia de este tipo de organización de la movilidad (Chatters 1987). Los restos alimenticios atestiguan mayor variabilidad (mamíferos terrestres y marinos, aves, moluscos y peces) propia de una dieta íntegra; y en casos específicos han podido ser atribuidos a estacionalidad estival (p.ej. mamíferos marinos neonatos y peces Cilus gilberti y Trachurus symmetricus que se acercan en la temporada como cardúmenes, Jackson et al. 1997). Las categorías de instrumental lítico que caracterizan a estos sitios corresponden fundamentalmente a instrumentos de molienda para el procesamiento de alimentos u otros recursos inorgánicos (Jackson 2004), puntas de proyectil y cuchillos bifaciales descartados por agotamiento de su vida útil y los desechos de su reavivado (Arroyo 2000; Jackson 2002). Las categorías bifaciales fueron confeccionadas sobre diversas materias primas alóctonas de alta calidad, factor que, en conjunto con lo restringido de su secuencia reductiva depositada, sugiere una manufactura distante y actividad de transporte de alguna intensidad, bajo una lógica de prolongación de sus vidas útiles (conservación).

Una visión integrada ha permitido entender a los dos tipos de sitios como producto de la acción de una misma población (p.ej. traslape de dataciones entre campamentos de distinta funcionalidad, Tabla 2, Figura 3a) y no como distintos grupos cohabitando un mismo espacio. La interpretación formulada por Jackson (2002) postularía dos fases diferenciadas de ocupación del litoral, con un primer acercamiento exploratorio con eventuales visitas antes de los $\sim 6.800$ años cal. a.p. y una posterior trashumancia reiterada. Este patrón implicó un sistema de movilidad a gran escala, el cual -al menos en Los Vilos- se manifestó en una organización logística muy pautada, sostenida por algunos investigadores como "óptima" para el asentamiento costero (Yesner 1980). La evaluación de estos postulados requerirá continuar con las investigaciones tanto en el litoral como en territorios interiores de ambas vertientes de la cordillera. Indicadores arqueológicos del litoral Pacífico registrados en la vertiente oriental de la Cordillera de los Andes respaldarían los supuestos (Gambier 1993).

A partir de los $\sim 4.000$ años cal. a.p., en contemporaneidad con los mencionados cambios climáticos, y hasta los 2.000 años cal. a.p. (Tabla 2) prevaleció un sistema de asentamientos y movilidad muy distinto, no obstante sea posible sostener una continuidad cultural (Méndez 2003). Los yacimientos habitacionales proliferan en el borde costero, a la vez que se minimizan localidades de tareas específicas (Méndez y Jackson 2004). Los campamentos crecen, se inicia la ocupación de sectores previamente no aprovechados, en conjunto con una frecuente reocupación de localidades. Su ubicación (Figura 3b) obedece a una lógica de obtención de los recursos que moviliza a la gente y sus campamentos residenciales hacia los sectores más propicios para el forrajeo (Méndez 2003; Méndez y Jackson 2004). Asimismo, en contraposición con el patrón anterior, estos grupos explotaron la totalidad de los recursos consumibles en el área, incluyéndose toda la disponibilidad de moluscos (bivalvos y gasterópodos de distintos micro-hábitat), equinodermos, crustáceos, mamíferos marinos y terrestres (en mayor variabilidad y frecuencia) y aves. Se plantea un uso continuo de la costa a lo largo del ciclo anual a partir de indicadores, tanto de estacionalidad estival (como los mencionados para el caso anterior) como invernal, es el caso de los guanacos (Lama guanicoe) que sólo habrían accedido durante estas temporadas a la costa.

Los conjuntos instrumentales, si bien poseen una fuerte raigambre en las tecnologías desarrolladas durante el momento anterior, poseen algunas diferencias. Entre ellas destaca el uso de recursos líticos de aprovisionamiento local inmediato y regional a lo largo del eje costero, lo que sugiere un conocimiento acabado de las fuentes secundarias de rocas aptas para la talla. Proliferan ampliamente las categorías instrumentales toscas de procesamiento y molienda, ahora representadas en todos los asentamientos, y son frecuentes las puntas de proyectil en la totalidad de sus etapas de manufactura y vida útil. Los contextos de descarte, en conjunto con las evidencias antes mencionadas, permiten pensar en un uso más intensivo del eje costero, selectividad que se vincularía -hipotéticamente- a la mayor capacidad de carga en la zona costera de Los Vilos que en territorios vecinos hacia el norte y sur (Báez 2000).

Una mayor intensidad de estadía en el espacio también se sugiere a partir de evidencias de influencia antrópica sobre el medio, como la mayor concentración de carbones en Ñagué I hacia los 3.200 años cal. a.p. (Maldonado y Villagrán 2002). No 
obstante el mayor grado de ocupación permanente del área, los cazadores recolectores se mantuvieron altamente móviles, cambiando de residencias con mayor frecuencia, aunque realizando desplazamientos más cortos. Esta conducta explica la redundancia en la ocupación de los asentamientos, manifiesta en la disrupción de rasgos estructurales y contextos con palimpsestos en densas estratigrafías (Méndez y Jackson 2004).

\section{Discusión y Comentarios Finales}

Convencionalmente, una correlación positiva entre cambios medioambientales y culturales se asume a modo de una situación de causa y efecto sin mayor cuestionamiento, aunque bien pueda deberse a concurrencia o coincidencia (Dincauze 2000). La discusión del modelo presentado intenta definir qué variables pueden ser entendidas bajo la primera lógica, y cuáles podrían explicarse alternativamente (p.ej. equifinalidad). En este sentido, se entiende que los sistemas sociales son multidimensionales, compuestos por entidades, sus relaciones y sus modificaciones en el tiempo (Dincauze 2000), constituyendo una realidad compleja, que no puede ser comprendida unívocamente (i.e. causalidad). Entender así los sistemas sociales genera muchas más preguntas que respuestas, dificultando llegar a conclusiones simples.

Por una parte, como seres humanos, nuestra experiencia ambiental ocurre a escala local y se encuentra mediada por el lenguaje y la oportunidad (Dincauze 2000). Por otro lado, la característica esencial de los cazadores recolectores es su ethos de la movilidad, percepción que valora el movimiento residencial, pese a que estén dadas las condiciones para su restricción (Kelly 1995). Entonces, cuando referimos a respuestas sociales o decisiones para paliar los problemas externos, al trabajar con conjuntos humanos no productores, el movimiento puede entenderse como dichas respuestas. Estructurar distintos tipos de movilidad constituye la base de las respuestas sociales al ambiente, aunque no sea la única (i.e. fluctuación en el número de integrantes de las bandas, métodos de control de la población, explotación de recursos antes despreciados o de menor retorno, etc.). No obstante, los grupos humanos no reaccionan de manera inmediata ni menos automática ante lo "extra-somático", sino que las respuestas están mediadas tanto por sus efectos sobre los recursos seleccionados como por las relaciones culturalmente establecidas con los mismos (percepción). Sería esperable entonces una adecuación desfasada o reconfiguración de los patrones, posterior a las manifestaciones de las modificaciones climáticas, aunque siempre con un correlato sobre las preferencias selectivas del momento anterior.

La primera respuesta social al cambio medio ambiental corresponde al modelo hipotético desarrollado por Jackson (2002), donde la ocupación estival de la costa de Los Vilos operaría a modo de un acceso "transitorio" a un área ecotonal (buffer zone). Su propósito central sería paliar el riesgo durante un pulso climático adverso (Lanata y Borrero 1994) mediante la obtención de recursos tradicionalmente percibidos como "de emergencia", como son los moluscos (Yesner 1980). Se encuentra sustentada sobre procedencia de materias primas foráneas, representación exclusiva de etapas terminales de reducción bifacial, indicadores de estacionalidad estival en los sitios, organización logística, y por tanto transitoria, del espacio. Ante el advenimiento de condiciones de humedad y mejora ambiental general, los viajes trasandinos son desincentivados, generándose una ocupación "permanente" del área, o la segunda respuesta. Esta se sustenta en los siguientes indicadores: uso de la totalidad de recursos -bióticos y abióticos- del litoral, la representación de distintas estaciones en los conjuntos de fauna, uso más intenso y redundante del espacio, la representación íntegra de las secuencias de reducción lítica en los campamentos y un aumento en más de cinco veces la cantidad de campamentos residenciales en comparación al momento anterior (en la cuantificación más conservadora).

Un primer cuestionamiento es que si las costas son tan productivas (Yesner 1980), con recursos confiables (Waselkov 1987), y utilizadas entre los 7.700 y 4.200 años cal. a.p. a modo de una zona de amortiguamiento económico para grupos interiores, por qué se dio un establecimiento permanentemente sólo posterior a los $\sim 4.000$ años cal. a.p. Como alternativas es posible plantear al menos dos líneas de reflexión. Primero, que el litoral no haya sido tan productivo durante el pulso climático árido y sólo pudo mantener a los grupos hasta el advenimiento de las nuevas condiciones externas. Vale decir, que la aridez afectó de manera tal, que en ambas vertientes de la cordillera, 
incluida la costa, no se lograban las condiciones para la subsistencia a lo largo del ciclo anual. O incluso más, la producción costera no superaría (o igualaría) a la interior, sino hasta el arribo de la humedad. En ese entendido, los grupos debieron, motivados por las condiciones medioambientales, estructurar sus respuestas por medio de una movilidad extendida.

Como segunda alternativa, es posible que la costa del área de estudio bien pudiera sostener poblaciones humanas permanentemente a lo largo de todo el Holoceno, y entonces las razones para no establecerse de forma definitiva obedecerían a factores culturales. Bajo este razonamiento, los cazadores recolectores trasandinos, motivados por las restricciones de recursos, iniciaron un proceso de exploración a nuevos territorios, aunque sin desvincularse de sus regiones originarias. De aceptar la segunda aproximación, implicaría que, si bien la costa era concebida como zona de amortiguación, factores intrínsecos no permitieron un traslado poblacional definitivo como respuesta. Bajo esta lógica podría entenderse una aproximación gradual hacia la costa, con posibles periodos entre $\sim 7.700-6.800, \sim 6.800-4.200$ (Jackson 2002) y 4.000-2.000 años cal. a.p. (Méndez 2003; Méndez y Jackson 2004), correspondiente a etapa exploratoria inicial con movimientos restringidos, acceso estacional estival ampliado y ocupación permanente, respectivamente (Méndez 2004).

Aún más, de aceptarse - para el caso- la aridización como incentivo para la explotación litoral, ello no da cuenta de por qué con el advenimiento de condiciones más benignas una población se radicó permanentemente -aunque sin dejar de ser móvil- en el litoral. Pese a la evidente contemporaneidad con las modificaciones climáticas, una situación de estricta causalidad no resulta del todo sostenible. Más parece que una integración de variados factores dio pie a la mayor permanencia en territorios previamente ocupados de manera menos intensa, como el caso del área litoral de Los Vilos.

Como interpretación planteamos que uno de estos factores corresponde a la mayor intensidad en la ocupación del espacio macrorregional (Norte Semiárido, Chile Central, vertiente oriental de los Andes), la que devino en la delimitación de territorios más circunscritos. Ello supone implícitamente un alza demográfica, aunque en ningún caso una saturación del espacio, sino más bien una recomposición en los circuitos de movilidad que limitó las posibilidades más ampliadas que caracterizaron momentos previos. Proponemos que este proceso pudo estar mediatizado a través de la fisión de bandas como método de regulación de la población al interior de las unidades residenciales.

Uno de los principales indicadores de esta nueva intensidad de uso del espacio se observa en la aparición de una mayor cantidad de yacimientos que documentan este momento tardío, incluso en sectores donde no se advierten ocupaciones previas. Lo anterior podría implicar un asentamiento paralelo en mayor cantidad de áreas, de lo cual sugerimos emergieron fenómenos de competencia por el espacio, por los recursos y, con toda probabilidad, un fenómeno de territorialidad previamente imperceptible o inexistente. Manifestación de ello en el área de Los Vilos se atestigua en los grandes montículos de conchas, producto del reiterado descarte intencional sobre un mismo sector, cuestión que interpretamos como marcadores espaciales en áreas de alto potencial biótico. Estos grandes conchales del Holoceno Tardío destacan visiblemente en el paisaje, evidenciando, por sobre todo, la necesidad de los grupos de manifestar su presencia en el espacio, extendiendo los alcances de su actuar cotidiano hacia el territorio.

Agradecimientos: El trabajo fue realizado con el apoyo de la Beca Proyecto MECESUP-UTA9903 en el marco de los estudios de maestría del primer autor en la Universidad Católica del Norte y Universidad de Tarapacá. Los sitios fueron en su mayoría investigados a través de los proyectos FONDECYT 91-0026, 1950372, 1990699 y en el marco de un estudio de impacto ambiental financiado por Minera Los Pelambres. Los permisos para trabajar numerosos sitios arqueológicos fueron otorgados por la familia Matte, a través del Sr. Alberto Alfonso, a quienes se agradece particularmente. Agradecemos especialmente al Dr. Lautaro Núñez y a dos revisores anónimos por sus valiosos comentarios al manuscrito original. 


\section{Referencias Citadas}

Arroyo, M.

2000 Estudio exploratorio de las cadenas operatorias de reducción lítica en el sitio LV-166 Dunas de Agua Amarilla, Comuna de Los Vilos. Actas del XIV Congreso Nacional de Arqueología Chilena, Tomo I: 659-691. Museo Regional de Atacama, Copiapó.

Artigas, D.

2001 Descifrando la superficie. Recolección superficial e intervención estratigráfica en un sitio Arcaico de la costa de Los Vilos. Boletín de la Sociedad Chilena de Arqueología 35/36:9-27.

Báez, P.

2000 Ambiente costero entre la desembocadura del río Choapa y Pichidangui: opinión crítica respecto a la biodiversidad marina. En Informe FONDECYT 1990699 - Año 1. Manuscrito conservado en la Biblioteca de CONICYT, Santiago.

Barrera, M. y C. Belmar

2000 Rescate del sitio arqueológico No 18 Paso Inferior Conchalí: Nuevas evidencias del Complejo Papudo en el Norte Semiárido. Valles 5/6:135-146.

Binford, L.

1980 Willow smoke and dogs' tails: hunter-gatherer settlement systems and archaeological site formation. American Antiquity 45:4-20.

Chatters, J.

1987 Hunter-gatherer adaptations and assemblage structure. Journal of Anthropological Archaeology 6:336-375.

Dincauze, D.

2000 Environmental Archaeology. Principles and Practice. Cambridge University Press, Cambridge.

Donoso, C.

1981 Investigación forestal y desarrollo. CONAF, Documento de Trabajo 30, Santiago.

Falabella, F., M. Planella y A. Pollastri

1991 Análisis de Oxígeno 18 en material malacológico de Chile Central. Actas del XI Congreso Nacional de Arqueología Chilena, Tomo I:105-121. Museo Nacional de Historia Natural y Sociedad Chilena de Arqueología, Santiago.

Fuenzalida, P.

1965 Geografía Económica de Chile. CORFO, Santiago.

Gambier, M.

1993 Prehistoria de San Juan. Editorial Fundación Universidad Nacional de San Juan, San Juan.

Grosjean, M., I. Cartajena, M. Geyh y L. Núñez

2003 From proxy data to paleoclimatic interpretation: the Mid-Holocene paradox of the Atacama Desert, northern Chile. Palaeogeography, Palaeoclimatology, Palaeoecology 3065:1-12.

Jackson, D.

2002 Cazadores y Recolectores del Holoceno Medio del Norte Semiárido de Chile. Tesis para optar al grado de Magíster en Arqueología, Departamento de Antropología, Universidad de Chile, Santiago.

2004 Los implementos de molienda en un campamento estacional del Holoceno Medio: implicancias funcionales y contextuales. Chungara Revista de Antropología Chilena, Volumen Especial: 95-103.

Jackson, D. y G. Ampuero

1993 Tecnología y recursos explotados en un campamento Arcaico de Los Vilos, Provincia del Choapa. Actas del XII
Congreso Nacional de Arqueología Chilena. Boletín del Museo Regional de la Araucanía 4:189-200.

Jackson, D., P. Báez y J. Arata

2004 Composición de conchales, estrategia de subsistencia y cambios paleoambientales en un asentamiento Arcaico, Norte Chico de Chile. Boletín de la Sociedad Chilena de Arqueología 37:37-48

Jackson, D., P. Báez, R. Seguel y J. Arata

1996 Campamento arcaico para la explotación intermareal: Significado del desconche local de moluscos. Valles 2:89-109.

Jackson, D. P. Báez y L. Vargas

1997 Secuencia ocupacional y adaptaciones durante el Arcaico en la Comuna de Los Vilos, Provincia de Choapa. Actas del XIII Congreso Nacional de Arqueología Chilena. Hombre y Desierto: Una Perspectiva Cultural 9:99-114.

Jenny, B., B. Valero-Garcés, R. Villa-Martínez, R. Urrutia, M. Geyh y H. Viet

2002 Early to Mid-Holocene aridity in Central Chile and the southern westerlies: The Laguna Aculeo record $\left(34^{\circ} \mathrm{S}\right)$. Quaternary Research 58:160-170.

Jerardino, A.

1995 Late Holocene neoglacial episodes in southern South America an southern Africa: A comparison. The Holocene 5:361-368.

Kelly, R.

1995 The Foraging Spectrum: Diversity in Hunter-Gatherer Lifeways. Smithsonian Institution Press, Washington y Londres.

Lanata, J. y L. Borrero

1994 Riesgo y arqueología. En Arqueología de CazadoresRecolectores. Límites, Casos y Aperturas, editado por J. Lanata y L. Borrero. Arqueología Contemporánea 5:129-143.

Lamy, F., D. Hebbeln y G. Wefer

1999 High resolution marine record of climatic change in mid-latitude Chile during the last 28,000 years based on terrigenous sediment parameters. Quaternary Research 51:83-93.

Maldonado, A. y C. Villagrán

2002 Paleoenvironmental changes in the semiarid coast of Chile $\left(\sim 32^{\circ} \mathrm{S}\right)$ during the last $6200 \mathrm{cal}$ years inferred from a swamp-forest pollen record. Quaternary Research 58:130138.

Mena, F.

1996 Evaluación crítica del paleoambiente de la Comuna de Los Vilos. En Informe de Avance Proyecto FONDECYT 1950372 - Año 1. Manuscrito conservado en la Biblioteca de CONICYT, Santiago.

Méndez, C.

2002a Cazadores recolectores costeros y sus contextos de tarea: Una visión desde el asentamiento holocénico temprano de Punta Penitente (LV. 014), Los Vilos. Chungara Revista de Antropología Chilena 34:153-166.

2002b Tecnología, Subsistencia y Movilidad en Punta Penitente (LV.014). Un Acercamiento Hacia los Patrones Conductuales de los Grupos de Cazadores Recolectores en el Litoral del Norte Semiárido. Memoria de título para optar al grado profesional de Arqueólogo, Departamento de Antropología, Universidad de Chile, Santiago.

2003 Orígenes del asentamiento holocénico tardío de cazadores recolectores en el litoral del Choapa: Ensayo para 
la generación de una hipótesis de investigación. Werken 4:43-58.

2004 Una sociedad de Cazadores Recolectores en Movimiento Durante la Primera Mitad del Holoceno Tardio en Los Vilos. Tesis para optar al grado de Magíster en antropología, Universidad Católica del Norte y Universidad de Tarapacá, San Pedro de Atacama.

Méndez, C. y D. Jackson

2004 Ocupaciones humanas del Holoceno Tardío en Los Vilos (IV Región, Chile): origen y características conductuales de la población local de cazadores recolectores de litoral. Chungara Revista de Antropología Chilena 36:279-293.

Méndez, C., J. Torres, P. López, F. Fernández y G. Rojas 2004 Excavación en área en yacimientos de cazadores recolectores: una propuesta metodológica. Werken 5:33-38.

Messerli, B., M. Grosjean, T. Hofer, L. Núñez y C. Pfister

2000 From nature-dominated to human-dominated environmental changes. Quaternary Science Reviews 19:459-479.

Montané, J.

1964 Fechamiento tentativo de las ocupaciones humanas en dos terrazas a lo largo del litoral chileno. Actas de Arqueología de Chile Central y Áreas Vecinas, pp. 109-124, Viña del Mar.

Núñez, L., J. Varela y R. Casamiquela

1987 Ocupación paleoindio en el centro norte de Chile: adaptación circumlacustre en las tierras bajas. Estudios Atacameños 8:142-185.

Núñez, L., J. Varela, R. Casamiquela y C. Villagrán

1994 Reconstrucción multidisciplinaria de la ocupación prehistórica de Quereo, centro de Chile. Latin American Antiquity 5:99-118.

Ota, Y. y R. Paskoff

1993 Holocene Deposits on the Coast of North-Central Chile: Radiocarbon Ages and Implications for Coastal Changes. Revista Geológica de Chile 20:25-32.

Paskoff, R.

1970 Recherches Géomorphologiques dans le Chili Semiaride. Biscaye Fréres Imprimeurs, Bordeaux.

Pimentel, G.

2001 Estrategias de subsistencia, funcionalidad y estacionalidad de un sitio Arcaico en el área de Dunas de Agua Amarilla, Provincia del Choapa. En Informe Proyecto FONDECYT 1990699 - Año 2. Manuscrito conservado en la Biblioteca de CONICYT, Santiago.

Reitz, E. y D. Sandweiss

2001 Environmental change at Ostra base camp, a Peruvian pre-ceramic site. Journal of Archaeological Science 28:1085-1100.

Román, A. y D. Jackson

1998 Dataciones por termoluminiscencia de rocas de fogones de asentamientos arcaicos, Provincia del Choapa. Chungara Revista de Antropología Chilena 30:41-48.
Sandweiss, D.

2003. Terminal Pleistocene through Mid-Holocene archaeological sites as paleoclimatic archives for the Peruvian coast. Palaeogeography, Palaeoclimatology, Palaeoecology 194:23-40.

Sandweiss, D., K. Maasch, R. Burger, J. Richardson III, H.

Rollins y A. Clement

2001 Variation in Holocene El Niño frequencies: Climate records and cultural consequences in ancient Peru. Geology 29:603-606

Sandweiss, D., J. Richardson III, E. Reitz, H. Rollins y K. Maasch 1996 Geoarchaeological evidence from Peru for a 5000 years B.P. onset El Niño. Science 273:1531-1533.

Seguel, R., D. Jackson, A. Rodríguez, X. Novoa, M. Henríquez,

P. Báez y D. Jackson

1995 Rescate de un asentamiento Diaguita costero: proposición de una estrategia de investigación y conservación. Proyecto DIBAM-FAI 94107. Manuscrito en posesión de los autores.

Stuiver, M., P. Reimer y T. Brazuiunas

1998 High-precision radiocarbon age calibration for terrestrial and marine simples. Radiocarbon 40:1127-1151.

Varela, J.

1976 Geología del cuaternario de la Laguna de Taguatagua (Prov. de O'Higgins). Actas del I Congreso Geológico 81-112, Santiago.

1981 Geología del cuaternario del área de Los Vilos-Ensenada El Negro (IV Región) y su relación con la existencia del bosque "relicto" de Quebrada Quereo. Comunicaciones 33:17-30.

Viet, $\mathrm{H}$.

1993 Upper Quaternary landscape and climate evolution in the Norte Chico (Northern Chile): An overview. Mountain Research Development 13:139-144.

Villagrán, C. y J. Varela

1990 Palynological evidence for increased aridity on the Central Chilean Coast during the Holocene. Quaternary Research 34:198-207.

Villa-Martínez, R. y C. Villagrán

1997 Historia vegetacional de bosques pantanosos de la costa de Chile central durante el Holoceno Medio y Tardío. Revista Chilena de Historia Natural 70:391-401.

Villa-Martínez, R., C. Villagrán y B. Jenny

2003 Pollen evidence for Late Holocene climate variability at Laguna Aculeo, Central Chile (lat. $34^{\circ} \mathrm{S}$ ). The Holocene 14:361-367.

Waselkov, G.

1987 Shellfish gathering and shell midden archaeology. En Advances in Archaeological Method and Theory 10, editado por M. Schiffer, pp. 257-310. Academic Press, New York. Yesner, D.

1980 Maritime hunter-gatherers: ecology and prehistory. Current Anthropology 21:727-735.

\section{Nota}

1 La única datación de la columna Ñagué III (5.400£40 a.p., Maldonado y Villagrán 2002) no se exhibe en la Figura 1. 